\title{
Apendicitis aguda neonatal: reporte de un caso
}

\author{
Neonatal acute appendicitis, case report
}

\author{
Nery Romero Rojas ${ }^{1}$, Luis Sánchez García ${ }^{2}$ \\ ${ }^{1}$ Instituto de Patología UNMSM \\ ${ }^{2}$ Hospital Nacional Docente de Cajamarca.
}

\begin{abstract}
Resumen
Introducción. La apendicitis aguda en neonatos es excepcionalmente rara. La mortalidad es muy alta y la perforación frecuente debido a problemas en el diagnóstico temprano en tanto el cuadro clínico es muy inespecifico. Se presenta un caso de apendicitis aguda no perforada en un neonato. Reporte de caso. Neonato de sexo femenino de 6 dias de edad y de $3170 \mathrm{~g}$ de peso, que desde el nacimiento rechazó la lactancia materna. Al tercer día presentó fiebre de $39^{\circ} \mathrm{C}$, distensión abdominal, vómitos múltiples y estreñimiento. Al examen físico se observó reptación en hemiabdomen superior y silencio abdominal. Una radiografía simple de abdomen mostró una cámara gástrica muy distendida; otros exámenes auxiliares fueron poco contributorios. Fue intervenida quirúrgicamente con el diagnóstico de oclusión intestinal, encontrándose apéndice cecal con múltiples adherencias laxas al íleo. Fue dada de alta a los pocos dias. El estudio histopatológico mostró apendicitis aguda gangrenosa no perforada. La apendicitis aguda debe ser considerada como parte del diagnóstico diferencial en la emergencia quirúrgica neonatal.
\end{abstract}

Palabras clave. Apendicitis Neonatal.

\section{Abstract}

Introduction. Acute appendicitis is a rare disease in newborns with a high mortality because of unspecific clinical features. We report a case of non-perforated acute appendicitis in a newborn at the San Bartolome Hospital in Lima. Case report. A female newborn weighing $3170 \mathrm{~g}$ in the first day repelled maternal lactation; at the third day, she presented $39^{\circ} \mathrm{C}$ fever, and later, abdominal distention, constipation, and abundant vomiting. The X-ray films showed a distended gastric camera and paralytic ileum; other auxiliary exams were inconclusive. She was operated with the diagnosis of intestinal obstruction; we found the ceccal appendix with multiple lax adhesions to the ileum. The pathologic study showed an acute not perforated gangrenous appendicitis. Appendicitis must be considered in the differential diagnosis of a neonatal surgical emergency.

Keywords. Neonatal Appendicitis.

An Fac med. 2016;77(4):421-22 / http://dx.doi.org/10.15381/anales.v77i4.12662

\section{INTRODUCCIÓN}

La apendicitis aguda es una entidad de jóvenes adultos y adolescentes, pero en neonatos es extremadamente rara; se estima una incidencia de $0,04 \%{ }^{(1)}$. Se menciona que Diess en $1903^{\text {(2) y }}$ Albrecht ${ }^{(3)}$ en 1905 publicaron los primeros casos. Karaman en una revisión de la literatura inglesa de 1901 al 2000 logró recolectar 141 casos, $75 \%$ eran hombres y $25 \%$ mujeres; en dicha casuística $52 \%$ fueron prematuros ${ }^{(1)}$.

La apendicitis neonatal puede presentarse como un proceso solitario ${ }^{(4)}$ o en asociación con diversas entidades, como enfermedad de Hirschprung (5-7), hernia inguinoescrptal ${ }^{(8,9)}$ encarcelada o no, cardiopatía congénita ${ }^{(10)}$, ente- ritis necrotizante ${ }^{(11)}$, fibrosis quística ${ }^{(6)}$, hernia umbilical ${ }^{(8)}$, neumonía, corioamnionitis ${ }^{(12)}$, mucormicosis ${ }^{(13)}$, atresia esofágica y fístula traqueoesofágica ${ }^{(14)}$, síndrome de Patau ${ }^{(15)}$, íleo meconial, perforación idiopática del colon, infecciones, entre otros. Así mismo, se ha señalado antecedentes de cesárea ${ }^{(16)}$ por DPP ${ }^{(3)} y$ preeclampsia ${ }^{(6)}$.

\section{REPORTE DEL CASO}

Se presenta el caso de un neonato de 6 días de edad, de sexo femenino que nació de parto normal con un peso de 3170 g. La niña fue producto de una segunda gestación cuya madre de 21 años tuvo un aborto anterior. La madre la trae al Hospital porque desde el naci- miento presentó rechazo a la lactancia materna; al tercer día de edad apareció fiebre de $39^{\circ} \mathrm{C}$ y al cuarto día estreñimiento y vómitos verdosos. $\mathrm{Al}$ examen clínico se encontró distensión abdominal, visualizándose reptación en hemiabdomen superior y a la auscultación silencio en hemiabdomen inferior. La radiografía simple de abdomen mostró una cámara gástrica muy distendida. Fue intervenida con el diagnóstico clínico de subobstrucción intestinal. En el acto quirúrgico se encontró asas ileales colapsadas y con múltiples adherencias alrededor del apéndice; este se encontraba muy congestivo y no se halló perforación. El estudio histopatológico mostró una lesión de apendicitis aguda gangrenosa (figura 1). Fue dada de alta a los 7 días, en buenas condiciones. 


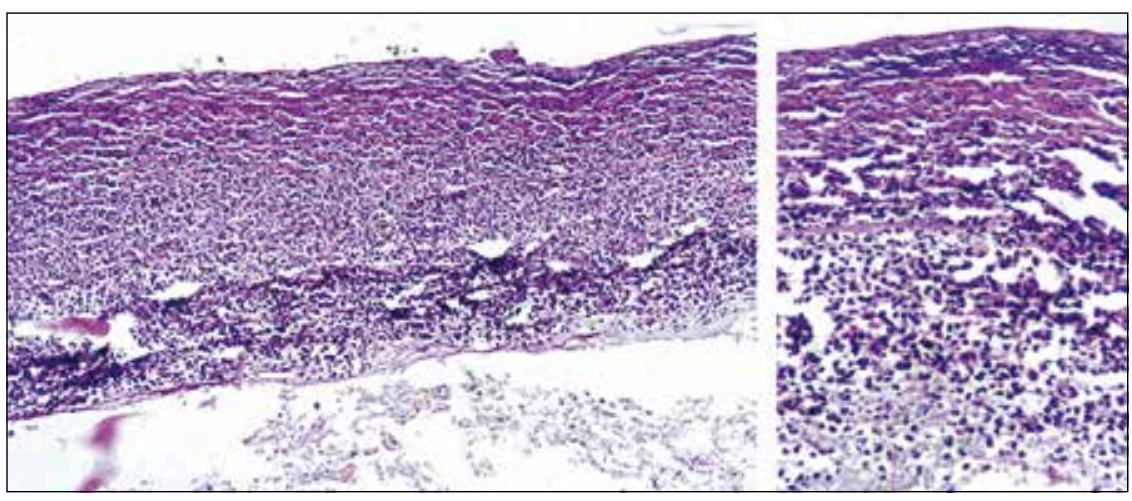

Figura 1. Pared apendicular necrótica e infiltrada por leucocitos polimorfonucleares; foto izquierda a 20X y foto derecha a 40X.

\section{DISCUSIÓN}

El diagnóstico de apendicitis neonatal es muy difícil y la mortalidad muy alta debido al cuadro clínico poco orientador ${ }^{(17)}$, siendo el síntoma más frecuente la distensión abdominal en $75 \%{ }^{(18)}$, que semeja un cuadro de obstrucción intestinal. Los exámenes auxiliares son poco contributorios ${ }^{(7,8)}$, porque no se toma en cuenta como parte del diagnóstico diferencial. A la fecha, no se ha reportado el diagnóstico clínico preoperatorio de apendicitis neonatal.

Ante la baja incidencia de apendicitis en neonatos, se ha propuesto diversos factores para explicarla, como:

1. La forma de embudo del apéndice (tipo fetal) con una amplia apertura en el ciego y un contorno relativamente recto;

2. La dieta líquida y falta de fecalitos;

3. La postura recostada del recién nacido; $y$,

4. La ausencia de hiperplasia linfoide apendicular ${ }^{(2,9,15,19)}$.

El caso que presentamos es el primero que se reporta en el Perú, con la característica de ser una niña a término sin perforación del apéndice y que salió recuperada del hospital.

Concluyendo, la apendicitis aguda neonatal es una enfermedad extremadamente rara, su mortalidad muy alta, presenta una sintomatología inespecífica que retarda el diagnóstico oportuno y un gran riesgo de perforación; se debe descartar otras entidades como el Hirschprung y considerarla en el diagnóstico diferencial de la emergencia quirúrgica neonatal

\section{REFERENCIAS BIBLIOGRÁFICAS}

1. Karaman A, Cavusoglu YH, Karaman I, Kakmak O. Seven cases of neonatal appendicitis with a review of the English language literature of the last century. Pediatr Surg Int. 2003;19:707-9.

2. Heydenrych JJ, Du Toit DF. Unusual presentations of acute appendicitis in the neonate. SA Med J. 1982;62(18):1003-5.

3. Massad M, Srouji M, Awdeh A, Slim M, Tamer M, Tabbara M. Neonatal appendicitis: case report and a revised review of the English literature. Z Kinderchir. 1986;41(4):241-3.

4. Gilbert-Barness E. Appendicitis. Potter's Pathology of the Fetus, Infant and Child, Second edition. 2007.

5. Arliss J, Holgersen O. Neonatal appendiceal perforation and Hirschsprung's disease. J Pediatr Surg. 1990;25:694-5.

6. Stiefel D, Stallmach T, Sacher P. Acute appendicitis in neonates: complication or morbus sui generis?. Pediatr Surg Int. 1998;14:122-3.
7. Gastrim U., Josephson S. Appediceal peritonitis and megacolon in the neonatal period. Acta Chir Scand. 1970;136:153-5.

8. Toledo EC, Castañón A, Gidi C, Ojeda E, Huesca JM. Hernia inguinoescrotal y apendicitis aguda en un recién nacido. Rev Mex Pediatr. 1999;66(1):21-2.

9. Raveenthiran V. Neonatal appendicitis: A Review of 24 cases with inguinoscrotal manifestation. J Neonatal Surg. 2015;4(2):1-5.

10. Trojanowski JQ, Gang DL, Goldblatt, Akins CW Fatal postoperative acute appendicitis in a neonate with congenital heart disease. J Pediatr Surg. 1981;16:85-6.

11. Rodríguez I, Garza A, González JM, Rodríguez JL, Garrocho H, Ortega M, Rodríguez R. Apendicitis neonatal perforada secundaria a enterocolitis necrosante. Bol Med Hosp Infant Mex. 1994;51:724-6.

12. Pressman A, Kawar B, Abend M, Steiner Z. Mogilner G. Acute perforated neonatal appendicitis associated with chorioamnionitis. Eur J Pediatr Surg. 2001;11:204-6.

13. Nichol PF, Corliss RF, Rajpal S, Helin M, Lund DP. Perforation of the appendix from intestinal mucormycosis in a neonate. J Pediatr Surg. 2004;39:1133-5.

14. Karaman A, Cavusoglu YH, Erdogan D. Appendiceal mass in a neonate after surgery for esophageal atresia and tracheosophageal fistula: report of a case. Surg. 2005;35(1):80-1

15. Pastore $\mathrm{V}$, Bartoli F. A Rare case of neonatal complicated appendicitis in a child with Patau's syndrome. Case Reports in Pediatrics. 2014. http:// dx.doi.org/10.1155/2014/671706.

16. Khan YA, Zia K, Saddal NS. Perforated neonatal appendicitis with pneumoperitoneum. APSP J Case Rep. 2013;4(2):21-2.

17. Messina M, Schiavone S, Meucci D, Garzi A. Acute perforated appendicitis in newborn. Pediatr Pediatr Med Chir. 1991;13(5):541-3.

18. Schwartz KL, Gilad E, Sigalet D, Yu W, Wong AL. Neonatal acute appendicitis: a proposed algorithm for timely diagnosis. J Ped Surg. 2011;46:2060-4.

19. Jahangiri $M$, Hosseinpour $M$, Jazayeri H, Mohammadzadeh M, Motaharizad D, Mirzadeh S. Perforated acute appendicitis in a pre-term neonate. Iran Red Crescent Med J. 2013;15(6):497-9.

Articulo recibido el 19 de abril de 2016 y aceptado para publicación el 7 de julio de 2016.

Conflicto de interés: Los autores declaran no tener conflicto de interés.

\section{Financiamiento: Propio de los autores.}

\section{Correspondencia:}

Dra. Nery Romero Rojas.

Dirección: Octavio Paz 193 Lima-34.

Correo electrónico: neryromerorojas@gmail.com 